\title{
Evaluation of the impact of linked color imaging for improving the visibility of colonic polyp
}

\author{
YASUYOSHI TANAKA $^{1 *}$, TAKUYA INOUE ${ }^{1,2^{*}}$, KAZUKI KAKIMOTO $^{1}$, KEI NAKAZAWA $^{1}$, \\ HIDEKI TAWA $^{1}$, YUKI HIRATA ${ }^{1}$, TOSHIHIKO OKADA ${ }^{1}$, SADAHARU NOUDA ${ }^{1}$, KEN KAWAKAMI ${ }^{1}$, \\ TOSHIHISA TAKEUCHI ${ }^{1}$, YUTARO EGASHIRA ${ }^{3}$ and KAZUHIDE HIGUCHI ${ }^{1}$ \\ ${ }^{1}$ Second Department of Internal Medicine, Osaka Medical College, Takatsuki, Osaka 569-8686; \\ ${ }^{2}$ Department of Gastroenterology, Inoue Gastroenterology and Endoscopy Clinic, Osaka 595-0072; \\ ${ }^{3}$ Department of Pathology, Osaka Medical College, Takatsuki, Osaka 569-8686, Japan
}

Received November 10, 2018; Accepted May 2, 2019

DOI: $10.3892 / \mathrm{ol} .2019 .10917$

\begin{abstract}
Linked color imaging (LCI) is a novel endoscopic system used to increase color contrast. As LCI does not decrease luminal brightness, it may improve the detection of colonic neoplasms. However, the extent to which LCI improves the visibility of colonic polyps has not yet been determined. Between December 2016 and May 2017, patients who received total colonoscopy were consecutively recruited into this retrospective, single-center study. For each polyp identified, images obtained from white light (WL) imaging, blue laser imaging (BLI), and LCI of the same lesion and its surrounding mucosa were evaluated. The color differences $(\Delta \mathrm{E})$ between each lesion and its surrounding mucosa in non-magnified images were computed quantitatively using the CIELAB color space, which defines color perception according to colorimetric values, and compared among $\mathrm{WL}$, BLI, LCI, and chromoendoscopy. The $\Delta \mathrm{E}$ between the vessel and non-vessel areas in magnified images was also assessed. Of the 64 patients who were incorporated into this study, non-magnified and magnified (x80) images from 113 and 95 polyps, respectively, were assessed. The $\Delta \mathrm{E}$ was intensified by LCI and chromoendoscopy compared with WL and BLI. The $\Delta \mathrm{E}$ of neoplastic lesions was also intensified by LCI. In magnified images, BLI and LCI significantly increased the $\Delta \mathrm{E}$ between the vessel and non-vessel areas compared with WL. Luminal brightness, indicated by $\mathrm{L}^{*}$, was not impaired by LCI; however, was reduced by BLI compared with WL and LCI. These results suggest that LCI enhanced the detection of colonic neoplasms without impairing luminal brightness. We
\end{abstract}

Correspondence to: Dr Kazuki Kakimoto, Second Department of Internal Medicine, Osaka Medical College, 2-7 Daigakumachi, Takatsuki, Osaka 569-8686, Japan

E-mail: in2098@osaka-med.ac.jp

${ }^{*}$ Contributed equally

Key words: linked color imaging, colonoscopy, adenoma, detection propose the routine use of LCI for colonic polyp detection and BLI for magnifying observations of colonic polyps detected by LCI.

\section{Introduction}

Colonoscopy is the criterion standard technique for identification of colonic neoplasia, and resection of colorectal adenomas is the most effective method for preventing colorectal cancer (1). Improved endoscopic procedures with higher rates of adenoma detection may improve the efficacy of colonoscopy for preventing cancer (1-3). Chromoendoscopy, which was implemented in the 1980s, has improved identification of small and flat colorectal lesions, and several randomized controlled trials have demonstrated significantly better rates of small neoplastic lesion detection by pan-colonic chromoendoscopy compared with conventional colonoscopy $(4,5)$. However, performing total colonic dye-spraying endoscopy requires a certain degree of endoscopic skills and longer extubation compared with conventional colonoscopy (6). The use of narrow-band imaging (NBI), which applies short-wavelength light to enabling detection of the absorbance spectrum of hemoglobin, during colonoscopy is expected to enhance adenoma detection without prolonging the extubation period (7). However, the ability of first-generation NBI systems to increase adenoma detection has not been demonstrated, considering that intestinal fluids such as bile appear red in color, like blood, and that the luminal brightness is lower in NBI than in conventional colonoscopy (8). To offset these constraints, blue laser imaging (BLI), which utilizes narrow-band laser light combined with white laser light, was developed. However, the brightness of BLI is still not adequate for detecting distant lesions (9).

Linked color imaging (LCI) is a novel endoscopic system developed by Fujifilm Co. that increases color contrast by utilizing short-wavelength narrow-band laser light combined with white laser light on the basis of BLI technology (9). Unlike BLI and NBI, the luminal brightness of LCI is not impaired compared with that of conventional colonoscopy, even at a distant view; consequently, LCI may improve the visibility of colonic neoplasms $(10,11)$. However, thus far, the 
extent to which LCI improves the visibility of colonic polyps has not been clarified quantitatively. Therefore, in this study, we evaluated the color differences between colonic polyps and their surrounding mucosa and compared the color differences among conventional colonoscopy, BLI, LCI, and chromoendoscopy.

\section{Patients and methods}

Endoscopic procedure. This was a retrospective single-center study. Between December 2016 and May 2017, all patients who received a total colonoscopy at the Inoue Gastroenterology and Endoscopy Clinic were recruited. The Ethics Committee of Osaka Medical College approved this study, and written informed consent was waived because of the retrospective design. For the endoscopic procedures, signed informed consent was obtained from the patients. The inclusion criteria were as follows: i) surveillance colonoscopy of known colorectal adenomas or cancer; ii) screening colonoscopy in patients with positive fecal occult blood; and iii) diagnostic colonoscopy in patients with symptoms, such as rectal bleeding, abdominal pain, and change in bowel habits. Patients with inadequate bowel preparation, colitis, and melanosis did not enter the study. For each polyp identified, pictures with three different color images (WL, BLI, and LCI) for the same lesion and from the same point of view were selected for evaluation. Among the selected pictures, those of chromoendoscopy for the same lesion and from the same point of view were also used for evaluation. In the case of magnified colonoscopic images, high magnification (x80) pictures with three different color images for the same lesion and from the same point of view were selected for evaluation. Colonoscopy was conducted using the LASEREO system (FUJIFILM) with an EC-L600ZP endoscope. Endoscopy was performed using the WL, BLI, and LCI modalities. All endoscopic images were stored in JPEG format. An experienced endoscopist (T.I.) performed the endoscopic procedures and selection of the images, and an experienced pathologist (Y.E.) examined all resected tissues.

Calculation of the color difference. The color difference $(\triangle E)$ was calculated using the CIELAB color space, a three-dimensional color space that comprises a black-white axis $\left(L^{*}\right)$, a red-green axis $\left(a^{*}\right)$, and a yellow-blue axis $\left(b^{*}\right)$ and that associates color perception with colorimetric values. $L^{*}$ is defined as lightness, $a^{*}$ as the red-green component, and $b^{*}$ as the yellow-blue component (12). $\Delta \mathrm{E}$ between a polyp (p) and the surrounding mucosa (b) was calculated according to the following formula: $\Delta \mathrm{E}_{\mathrm{pb}}=\sqrt{\left(L_{p}-L_{b}\right)^{2}+\left(a_{p}-a_{b}\right)^{2}+\left(b_{p}-b_{b}\right)^{2}}$. According to Sato et $a l$, we determined the $L^{*} a^{*} b^{*}$ values (13). Briefly, this was done as follows: i) the corresponding regions [for non-magnified images, the polyp and surrounding mucosa, 64 pixels each (Fig. 1), and for magnified images, the vessel and non-vessel areas, 9 pixels each (Fig. 2)] were selected on WL, BLI, and LCI images using Adobe Photoshop Elements 15; ii) the median RGB value was determined; and iii) the $L^{*} a^{*} b^{*}$ value was calculated from the average of the RGB values.

Statistical analysis. Statistical analyses were conducted using Stat View software, version 5.0 (SAS Institute, Inc.). All data are expressed as means \pm standard deviation. One-way
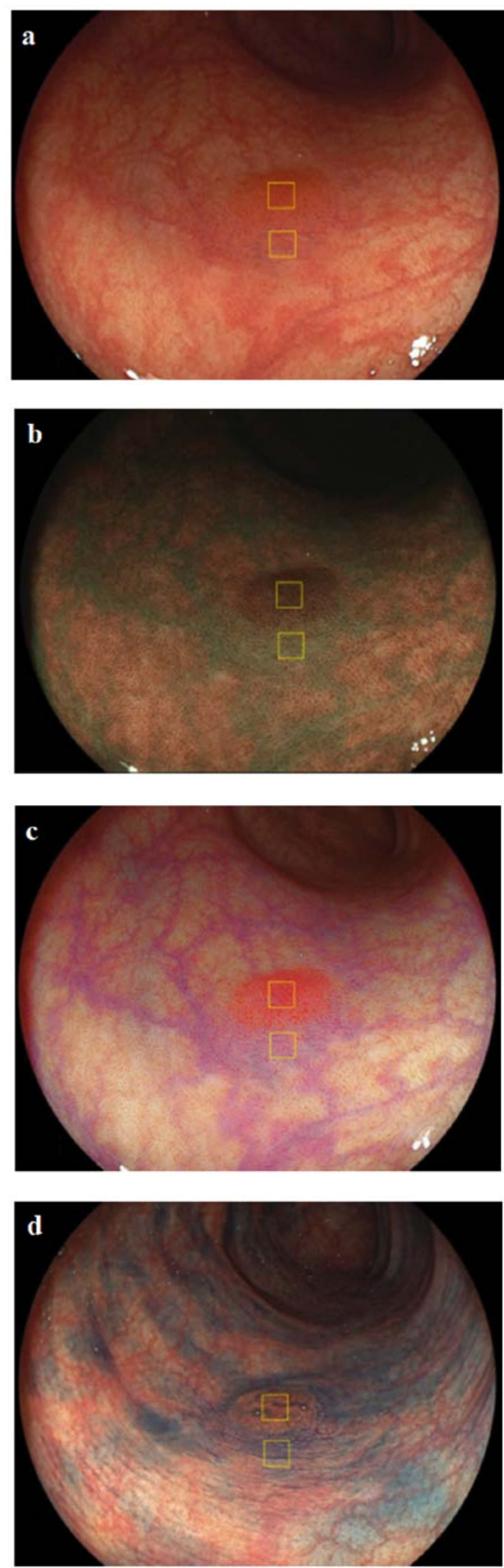

Figure 1. Selection of points for assessment of color differences in non-magnified colonoscopic images. Yellow square areas consisting of 64 pixels (8x8 pixels) show the same regions of the same polyps and their surrounding mucosa. (a) WL, (b) BLI, (c) LCI. (d) Chromoendoscopy. WL, white light; BLI, blue laser imaging; LCI, Linked color imaging. 

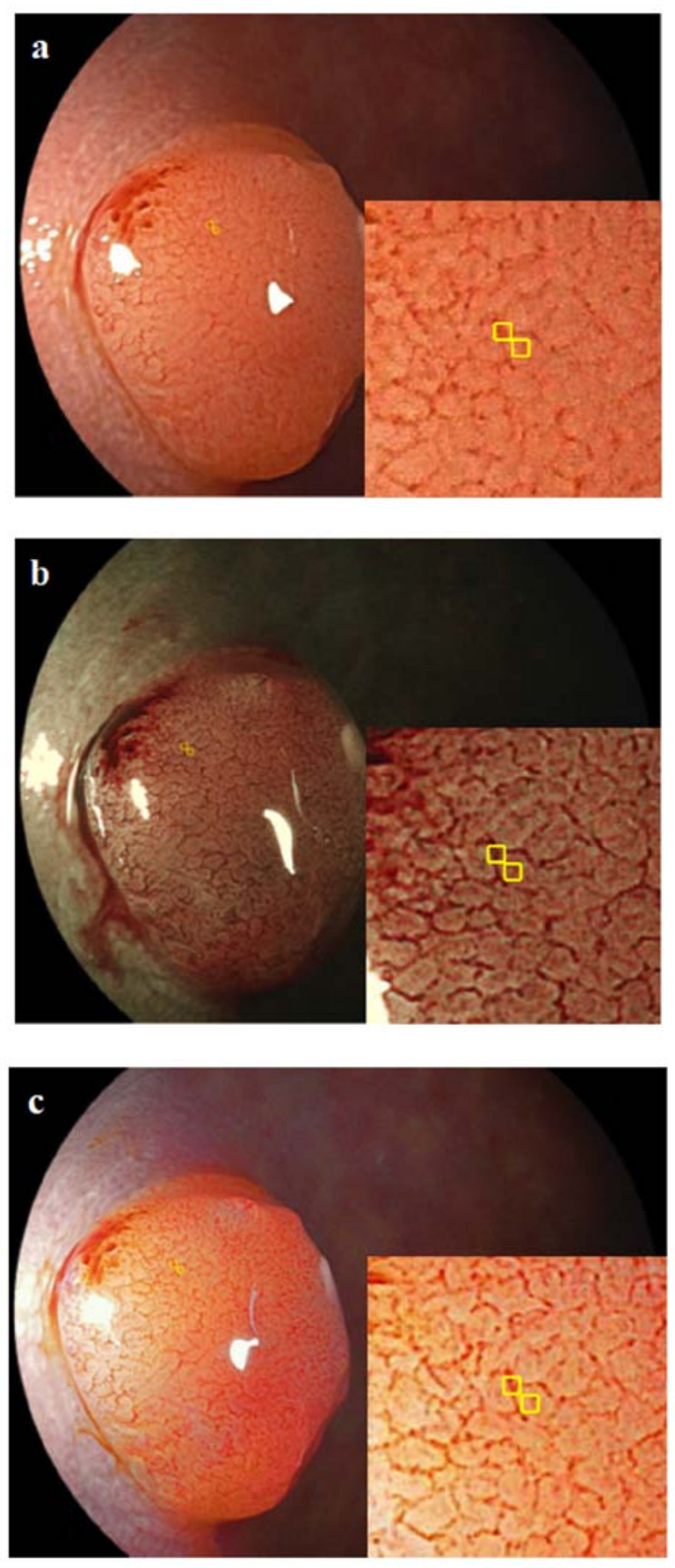

Figure 2. Selection of points for assessment of color differences in magnified colonoscopic images. Yellow square areas consisting of 9 pixels $(3 \times 3$ pixels) show the same vessel and non-vessel areas of the same polyp. (a) WL, (b) BLI, (c) LCI. WL, white light; BLI, blue laser imaging; LCI, Linked color imaging.

analysis of variance was performed for multiple comparisons, followed by Fisher's exact test. P-values of less than 0.05 were considered to be statistically significant.

\section{Results}

Color differences in non-magnified images. From December 2016 to May 2017, 64 patients with 114 polyps were enrolled in this study. Among these polyps, 113 (64 patients) and 95 (53 patients) were assessed for color differences in
Table I. Baselines characteristics of the patients and polyps.

\begin{tabular}{lc}
\hline Characteristic & Value \\
\hline Number of patients & 64 \\
Sex, male/female & $39 / 25$ \\
Age, years, mean \pm SD & $59.8 \pm 13.5$ \\
Number of polyps & 114 \\
Polyp size, mm, mean \pm SD & $7.6 \pm 5.0$ \\
Number of non-magnified images, & $113: 113: 113: 53$ \\
(WL:BLI:LCI:Chr), $n$ & \\
Number of magnified images, & $95: 95: 95$ \\
WL:BLI:LCI, $n$ & \\
Polyp location, right-sided:left-sided: & $52: 40: 22$, \\
rectum, $n(\%)$ & $(45.6: 35.1: 19.3)$ \\
Pathological diagnosis, neoplastic: & $99: 15,(86.8: 13.2)$ \\
non-neoplastic, $n(\%)$ & \\
\hline
\end{tabular}

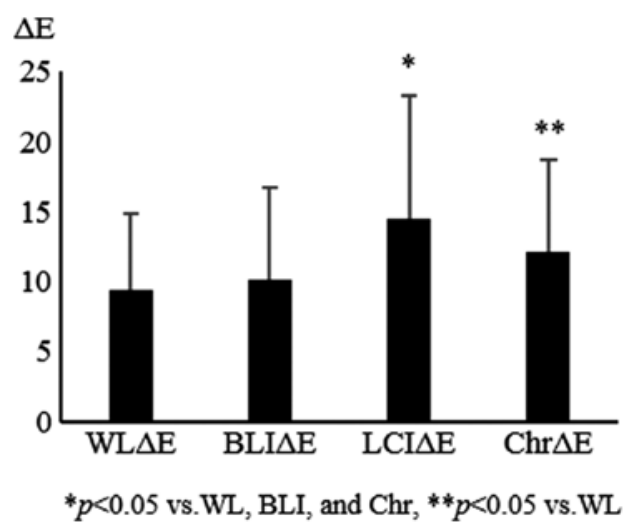

Figure 3. Overall $\Delta \mathrm{E}$. LCI and chromoendoscopy significantly increased the $\triangle \mathrm{E}$ between colonic polyps and their surrounding mucosa compared with WL and BLI. "P<0.05 vs. WL, BLI, and Chromoendoscopy, ${ }^{* *} \mathrm{P}<0.05$ vs. WL. $\mathrm{WL}$, white light; BLI, blue laser imaging; LCI, Linked color imaging; $\Delta \mathrm{E}$, color differences.

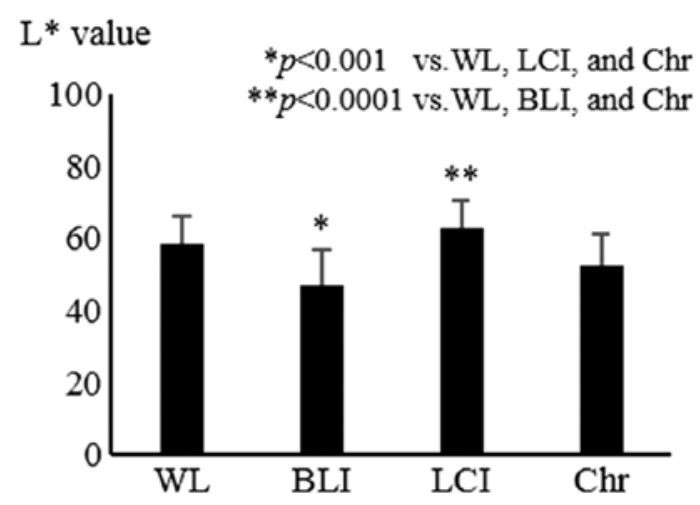

Figure 4. Background $L^{*}$. $L^{*}$ represents the luminal brightness. The $L^{*}$ of BLI was significantly decreased compared with that of WL and LCI. However, LCI did not impair the luminal brightness. "P<0.001 vs. WL, LCI, and Chromoendoscopy, ${ }^{* *} \mathrm{P}<0.0001$ vs. WL, BLI, and Chr. WL, white light; BLI, blue laser imaging; LCI, Linked color imaging.

non-magnified and magnified (x80) images, respectively (Table I). The overall $\Delta \mathrm{E}$ was significantly increased with 

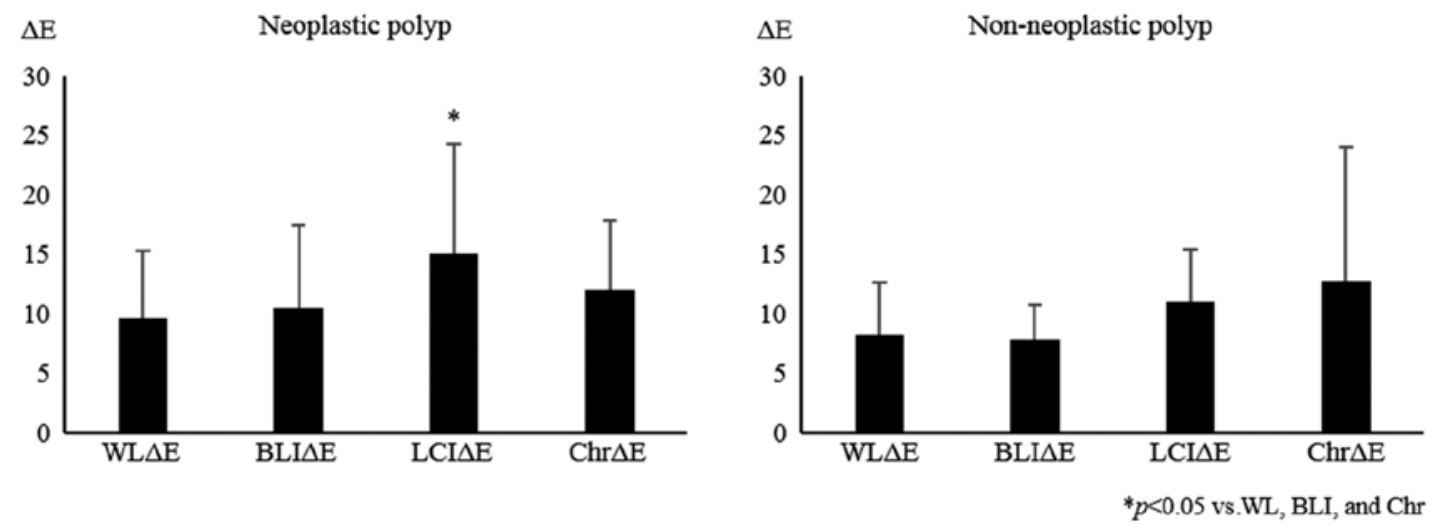

Figure 5. Neoplastic and non-neoplastic $\Delta \mathrm{E}$. In particular, LCI increased the $\Delta \mathrm{E}$ of neoplastic lesions (adenoma and cancer). ${ }^{*} \mathrm{P}<0.05 \mathrm{vs}$. WL, BLI, and Chromoendoscopy. WL, white light; BLI, blue laser imaging; LCI, Linked color imaging; $\Delta \mathrm{E}$, color differences.
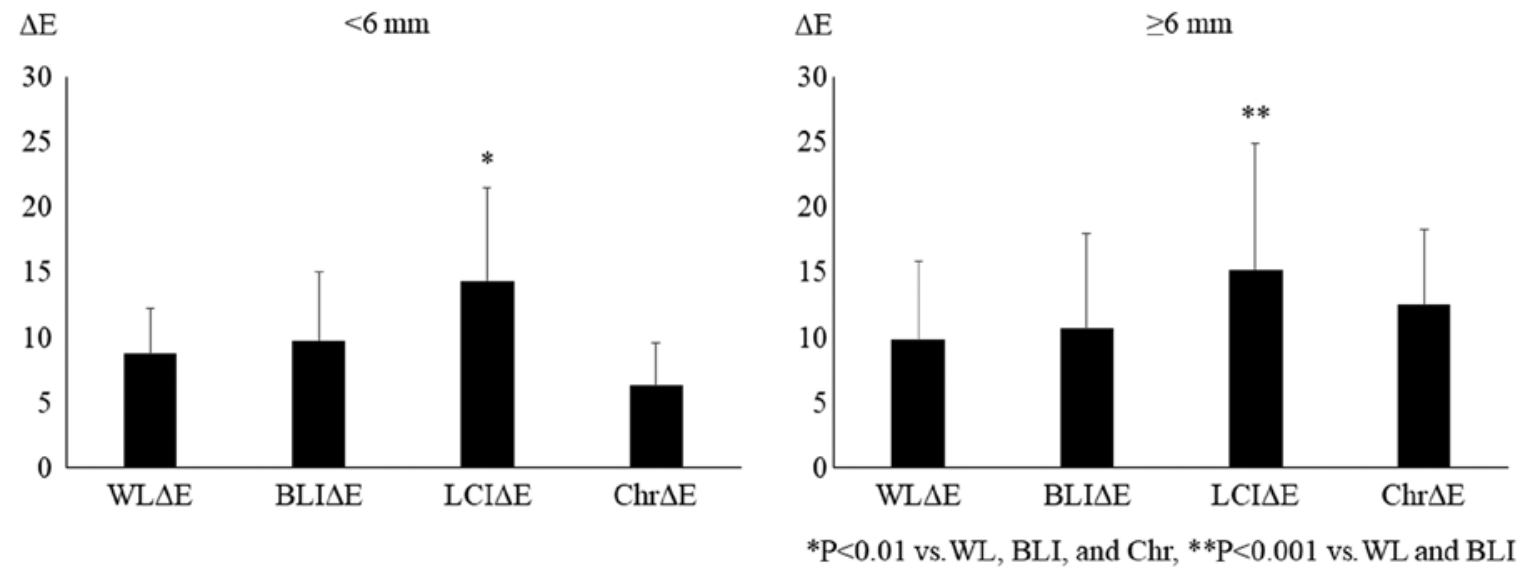

Figure 6. Size of neoplastic lesions and $\Delta \mathrm{E}$. LCI increased the $\Delta \mathrm{E}$ of neoplastic lesions regardless of the size. ${ }^{*} \mathrm{P}<0.01 \mathrm{vs}$. WL, BLI, and Chr, ${ }^{* *} \mathrm{P}<0.001 \mathrm{vs} . \mathrm{WL}$ and BLI. WL, white light; BLI, blue laser imaging; LCI, Linked color imaging; $\Delta \mathrm{E}$, color differences.

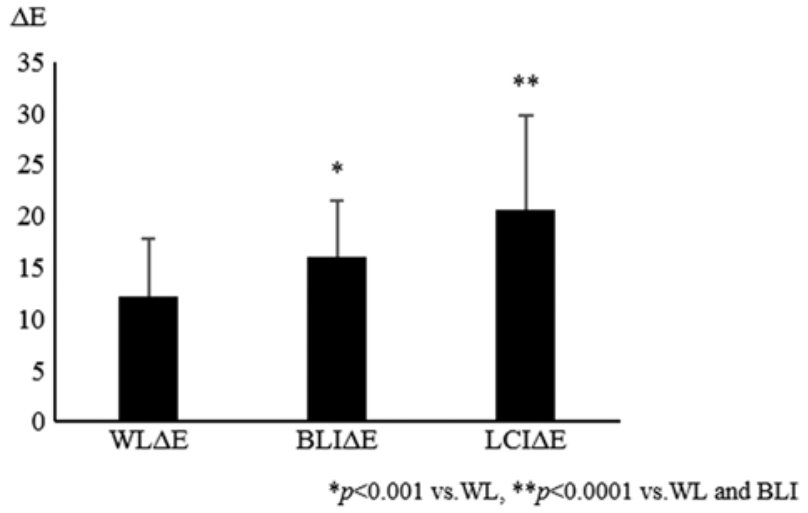

Figure 7. Color difference in magnified images. LCI and BLI significantly enhanced the $\Delta \mathrm{E}$ between the vessel and non-vessel areas on the surface of the neoplastic polyps. ${ }^{*} \mathrm{P}<0.001$ vs. WL, ${ }^{* *} \mathrm{P}<0.0001$ vs. WL and BLI. WL, white light; BLI, blue laser imaging; LCI, Linked color imaging.

LCI and chromoendoscopy compared with WL and BLI. The $\Delta \mathrm{E}$ values were $11.0 \pm 6.6,10.7 \pm 7.0,15.1 \pm 9.3$, and $14.8 \pm 7.2$ for WL, BLI, LCI, and chromoendoscopy, respectively (Fig. 3). $L^{*}$, which represents the lightness of a color, was significantly reduced by BLI compared with WL, LCI, and chromoendoscopy $(54.8 \pm 8.4,47.7 \pm 9.7,58.5 \pm 8.8$, and $51.7 \pm 9.4$ for $\mathrm{WL}$,
BLI, LCI, and chromoendoscopy, respectively) (Fig. 4). The $\Delta \mathrm{E}$ values of all neoplastic lesions (adenoma and cancer) were increased by LCI compared with WL, BLI, and chromoendoscopy. On the other hand, the $\Delta \mathrm{E}$ values of the non-neoplastic lesions were enhanced by chromoendoscopy compared with WL, BLI, and LCI, although the differences were not significant (Fig. 5). Regarding the effect of lesion size, LCI increased the $\Delta \mathrm{E}$ of neoplastic polyps in not only large (more than $6 \mathrm{~mm}$ in diameter) but also small (less than $6 \mathrm{~mm}$ ) lesions (Fig. 6). These findings suggest that LCI enhances the visibility of polyps, especially neoplastic polyps, even though they are small lesions, without impairing the lightness of the color.

Color differences in magnified images. Using magnified pictures of polyps, we calculated color differences between the vessel and non-vessel areas in the WL, BLI, and LCI images. The $\Delta \mathrm{E}$ was $12.2 \pm 5.6,16.1 \pm 5.5$, and $20.6 \pm 9.2$ for $\mathrm{WL}, \mathrm{BLI}$, and LCI, respectively. Overall, $\Delta \mathrm{E}$ was significantly increased in BLI and LCI images compared with that in WL images. BLI and LCI also had a significantly increased $\triangle \mathrm{E}$ in magnified images of neoplastic polyps (Fig. 7). These results indicate that BLI and LCI are useful in magnifying colonoscopy for diagnosis of colonic polyps. 


\section{Discussion}

To our knowledge, this is the first reported quantitative evaluation of the degree to which LCI enhances colonic polyp visibility. The results of this study revealed that LCI, compared with WL and BLI, significantly increased the color difference between polyps and their corresponding surrounding mucosa, without impairing luminal brightness. In particular, LCI significantly enhanced the visibility of neoplastic lesions, regardless of their size, but not non-neoplastic lesions, such as hyperplastic polyps and sessile serrated adenoma/polyps (SSA/P).

Considering that resection of polyps using colonoscopy has been reported to prevent the occurrence of colorectal cancer dramatically, by $76-90 \%$, colonoscopy is considered to be the most effective tool for colon cancer prevention (2). However, $17-24 \%$ of adenomas may be missed during colonoscopic examination $(13,14)$. Therefore, improved endoscopic methods that can accurately detect adenomas have been sought. Thus far, pan-colonic chromoendoscopy has been reported to increase the detection of small neoplastic lesions significantly, with significantly prolonged extubation times $(6,15,16)$. Since the NBI system was proposed for use during colonoscopy, several studies have been conducted to investigate the effect of $\mathrm{NBI}$ on improving the detection of colonic polyps (8). However, in these studies using first-generation NBI, it was not shown that NBI improves the adenoma detection rate compared with WL endoscopy (8). The reasons why these studies failed are considered to be related to the characteristics of NBI. For example, on NBI colonoscopy, intestinal fluids such as bile appear reddish, like blood, and the luminal brightness is reduced compared with that of conventional colonoscopy (7). Recently, a new-generation NBI system, which produces a twofold brighter image than the previous system, was reported to improve colonic polyp visibility and detection (17). Another novel image enhanced endoscopy (IEE) system, BLI, which enables one to obtain brighter images similar to new-generation NBI via the use of two different lasers creating white light illumination and short wavelength narrow-band light observation, was also revealed to improve the visibility of colorectal polyps $(18,19)$. However, thus far, the extent to which these IEE systems improve the visibility of colonic polyps has not been evaluated quantitatively.

Interestingly, in this study, LCI, but not BLI, quantitatively improved the visibility of colonic polyps; the corresponding surrounding mucosa was darker using BLI compared with that using WL and LCI. These results suggest that BLI still cannot provide enough luminal brightness to improve the color difference between colonic polyps and their corresponding surrounding mucosa, even though BLI creates brighter images than the old-generation IEE system. Therefore, we suggest that LCI is most suitable for routine colonoscopic examination for colonic polyp detection.

In the case of magnifying observation, BLI and LCI provided a significant color difference between the vessel and non-vessel (surface) area. It is well known that pit pattern analysis via magnifying chromoendoscopy is an accurate diagnostic method for the differentiation of colorectal lesions. Recently, several studies found that BLI magnification is accurate enough to diagnose most colorectal polyps $(20,21)$. Thus, the results regarding magnified images using BLI in this study confirm the results of those previous reports. We suggest that LCI is also useful for magnifying observation.

This study has some limitations. First, obtaining exact same timing of the three different color images (WL, BLI, and LCI) for the same lesion is impossible because of time lags. Second, this was a single-center retrospective study, and the number of samples was small. Moreover, all endoscopic procedures and selection of the images were performed by the same endoscopist. Third, because BLI uses a narrow wavelength range to provide information about microvessels, the color difference calculation using the CIELAB color space might not reflect the visibility of colonic polyps exactly. Therefore, a larger sample size may be required to determine the efficacy of LCI in improving the visibility of non-neoplastic polyps including SSA/P.

In conclusion, LCI significantly improved the visibility of colonic polyps irrespective of the size of the lesion, without impairing the brightness of the color, and LCI and BLI significantly improved the color differences in the magnified images of neoplastic polyps. These outcomes support the routine use of LCI for colonic polyp detection and of BLI for improving magnifying observations of the colonic polyps detected by LCI. In this study, LCI did not influence color differences in non-neoplastic lesions, such as hyperplastic polyps and SSA/P. Considering that SSA/P was recently recognized to have a similar malignant potential as that of traditional adenoma, further investigation is needed.

\section{Acknowledgements}

This abstract was presented at the 2018 Digestive Disease Week in Washington, DC, USA and was published in Gastrointestinal Endoscopy 2018; 87 (6S): AB485.

\section{Funding}

No funding was received.

\section{Availability of data and materials}

The datasets used and/or analyzed during the current study are available from the corresponding author on reasonable request.

\section{Authors' contributions}

YT performed the data collection and procedures; TI and KaK wrote the manuscript and analyzed the data. TI also contributed to drafting conception and design; KN, HT, YH, TO, SN, $\mathrm{KeK}$ and TT participated in collecting the data; YE and $\mathrm{KH}$ contributed to the interpretation of the data and supervised the study. All authors have read and approved the final manuscript.

\section{Ethics approval and consent to participate}

The present study was approved by the Ethics Review Committee of Osaka Medical College (approval no. 2429). Due to the retrospective design of the current study and patient anonymization, the review board determined that informed consent was not required. 


\section{Patient consent for publication}

Not applicable.

\section{Competing interests}

The authors declare that they have no competing interests.

\section{References}

1. Winawer SJ and Zauber AG: Colonoscopic polypectomy and the incidence of colorectal cancer. Gut 48: 753-754, 2001.

2. Winawer SJ, Zauber AG, Ho MN, O'Brien MJ, Gottlieb LS, Sternberg SS, Waye JD, Schapiro M, Bond JH, Panish JF, et al: Prevention of colorectal cancer by colonoscopic polypectomy. The national polyp study workgroup. N Engl J Med 329: 1977-1981, 1993

3. Kaminski MF, Wieszczy P, Rupinski M, Wojciechowska U, Didkowska J, Kraszewska E, Kobiela J, Franczyk R, Rupinska M, Kocot B, et al: Increased rate of adenoma detection associates with reduced risk of colorectal cancer and death. Gastroenterology 153: 98-105, 2017.

4. Tada M, Katoh S, Kohli Y and Kawai K: On the dye spraying method in colonofiberscopy. Endoscopy 8: 70-74, 1977.

5. Tada M and Kawai K: Research with the endoscope: New techniques using magnification and chromoscopy. Clin Gastroenterol 15: 417-437, 1986.

6. Brooker JC, Saunders BP, Shah SG, Thapar CJ, Thomas HJ, Atkin WS, Cardwell CR and Williams CB: Total colonic dye-spray increases the detection of diminutive adenomas during routine colonoscopy: A randomized controlled trial. Gastrointest Endosc 56: 333-338, 2002.

7. Inoue T, Murano M, Murano N, Kuramoto T, Kawakami K, Abe Y, Morita E, Toshina K, Hoshiro H, Egashira Y, et al: Comparative study of conventional colonoscopy and pan-colonic narrow-band imaging system in the detection of neoplastic colonic polyps: A randomized, controlled trial. J Gastroenterol 43: 45-50, 2008

8. Ng SC and Lau JY: Narrow-band imaging in the colon: Limitations and potentials. J Gastroenterol Hepatol 26: 1589-1596, 2011.

9. Okada M, Sakamoto H, Takezawa T, Hayashi Y, Sunada K, Lefor AK and Yamamoto $\mathrm{H}$ : Laterally spreading tumor of the rectum delineated with linked color imaging technology. Clin Endosc 49: 207-208, 2016.

10. Yoshida N, Naito Y, Murakami T, Hirose R, Ogiso K, Inada Y, Dohi O, Kamada K, Uchiyama K, Handa O, et al: Linked color imaging improves the visibility of colorectal polyps: A video study. Endosc Int Open 5: E518-E525, 2017.
11. Min M, Deng P, Zhang W, Sun X, Liu Y and Nong B: Comparison of linked color imaging and white-light colonoscopy for detection of colorectal polyps: A multicenter, randomized, crossover trial. Gastrointest Endosc 86: 724-730, 2017.

12. Sato Y, Sagawa T, Hirakawa M, Ohnuma H, Osuga T, Okagawa Y, Tamura F, Horiguchi H, Takada K, Hayashi T, et al: Clinical utility of capsule endoscopy with flexible spectral imaging color enhancement for diagnosis of small bowel lesions. Endosc Int Open 2: E80-E87, 2014.

13. Rex DK, Cutler CS, Lemmel GT, Rahmani EY, Clark DW, Helper DJ, Lehman GA and Mark DG: Colonoscopic miss rates of adenomas determined by back-to-back colonoscopies. Gastroenterology 112: 24-28, 1997.

14. Bensen S, Mott LA, Dain B, Rothstein R and Baron J: The colonoscopic miss rate and true one-year recurrence of colorectal neoplastic polyps. Polyp prevention study group. Am J Gastroenterol 94: 194-199, 1999.

15. Hurlstone DP, Cross SS, Slater R, Sanders DS and Brown S: Detecting diminutive colorectal lesions at colonoscopy: A randomised controlled trial of pan-colonic versus targeted chromoscopy. Gut 53: 376-380, 2004.

16. Le Rhun M, Coron E, Parlier D, Nguyen JM, Canard JM, Alamdari A, Sautereau D, Chaussade S and Galmiche JP: High resolution colonoscopy with chromoscopy versus standard colonoscopy for the detection of colonic neoplasia: A randomized study. Clin Gastroenterol Hepatol 4: 349-354, 2006.

17. Ogiso K, Yoshida N, Siah KT, Kitae H, Murakami T, Hirose R, Inada Y, Dohi O, Okayama T, Kamada K, et al: New-generation narrow band imaging improves visibility of polyps: A colonoscopy video evaluation study. J Gastroenterol 51: 883-890, 2016.

18. Yoshida N, Hisabe T, Hirose R, Ogiso K, Inada Y, Konishi H, Yagi N, Naito Y, Aomi Y, Ninomiya K, et al: Improvement in the visibility of colorectal polyps by using blue laser imaging (with video). Gastrointest Endosc 82: 542-549, 2015.

19. Togashi K, Nemoto D, Utano K, Isohata N, Kumamoto K, Endo S and Lefor AK: Blue laser imaging endoscopy system for the early detection and characterization of colorectal lesions: A guide for the endoscopist. Therap Adv Gastroenterol 9: 50-56, 2016.

20. Nakano A, Hirooka Y, Yamamura T, Watanabe O, Nakamura M, Funasaka K, Ohno E, Kawashima H, Miyahara R and Goto H: Comparison of the diagnostic ability of blue laser imaging magnification versus pit pattern analysis for colorectal polyps. Endosc Int Open 5: E224-E231, 2017.

21. Yoshida N, Yagi N, Inada Y, Kugai M, Okayama T, Kamada K, Katada K, Uchiyama K, Ishikawa T, Handa O, et al: Ability of a novel blue laser imaging system for the diagnosis of colorectal polyps. Dig Endosc 26: 250-258, 2014. 